\title{
Nucleon sigma term and strange quark content in 2+1-flavor QCD with dynamical overlap fermions
}

\author{
H. Ohki ${ }^{* a, b}$, S. Aoki ${ }^{c, d}$ H. Fukaya ${ }^{e}$, S. Hashimoto ${ }^{f, g}$, T. Kaneko ${ }^{f, g}$ H. Matsufuru ${ }^{f}$, \\ J. Noaki ${ }^{f}$, T. Onogi ${ }^{h}$, E. Shintani ${ }^{h}$, N. Yamada ${ }^{f, g}$ (for JLQCD collaboration) \\ ${ }^{a}$ Department of Physics, Kyoto University, Kyoto 606-8501, Japan, \\ ${ }^{b}$ Yukawa Institute for Theoretical Physics, Kyoto University, Kyoto 606-8502, Japan, \\ ${ }^{c}$ High Energy Accelerator Research Center, Brookhaven National Laboratory, Upton, New York \\ 11973, USA \\ ${ }^{d}$ School of High Energy Accelerator Science, The Graduate University for Advanced \\ studies(Sokendai), Ibaraki 305-0801, Japan \\ ${ }^{e}$ Department of Physics, Nagoya University, Nagoya 464-8602, Japan \\ ${ }^{f}$ High Energy Accelerator Research Organization (KEK), Tsukuba 305-0801, Japan, \\ ${ }^{g}$ School of High Energy Accelerator Science, The Graduate University for Advanced Studies \\ (Sokendai), Tsukuba 305-0801, Japan, \\ ${ }^{h}$ Department Physics, Osaka University, Toyonaka, Osaka 560-0043, Japan \\ E-mail: ohki@yukawa.kyoto-u.ac.jp
}

We study the sigma term and the strange quark content of nucleon in 2+1-flavor QCD with dynamical overlap fermions. We analyze the lattice data of nucleon mass taken at two different strange quark masses with five values of up and down quark masses each. Using the reweighting technique, we study the strange quark mass dependence of the nucleon and extract the strange quark content.

The XXVII International Symposium on Lattice Field Theory - LAT2009

July 26-31 2009

Peking University, Beijing, China

\footnotetext{
* Speaker.
} 


\section{Introduction}

Nucleon sigma term $\sigma_{\pi N}$ is given by a scalar form factor of nucleon at zero recoil. While up and down quarks contribute to $\sigma_{\pi N}$ both as valence and sea quarks, strange quark appears only as a sea quark contribution. As a measure of the strange quark content of the nucleon, the $y$ or $f_{T_{s}}$ parameters are commonly introduced. These parameters are defined as

$$
\sigma_{\pi N}=m_{u d}\langle N|\bar{u} u+\bar{d} d| N\rangle, \quad y \equiv \frac{2\langle N|\bar{s} s| N\rangle}{\langle N|\bar{u} u+\bar{d} d| N\rangle}, \quad f_{T_{s}}=\frac{m_{s}\langle N|\bar{s} s| N\rangle}{M_{N}} .
$$

The $y$ parameter plays an important role to determine the detection rate of possible neutralino dark matter in the supersymmetric extension of the Standard Model [1, 2].

Using lattice QCD, one can calculate the nucleon sigma term directly. Furthermore, it is possible to determine the valence and sea quark contributions separately. Previous lattice studies of the nucleon sigma term and strange quark content have been done within the quenched approximation [3, 4], or a two-flavor QCD calculation [5, 6]. Recent studies use 2+1 flavor QCD [7].

There was an apparent puzzle in these results: the strange quark content is unnaturally large compared to the up and down contributions that contain the connected diagrams too. Concerning this problem, it was pointed out that using the Wilson-type fermions, the sea quark mass dependence of the additive mass renormalization and lattice spacing can give rise to a significant lattice artifacts in the sea quark content [8]. Our previous study from a two-flavor QCD simulation using the overlap fermion removed this problem by explicitly maintaining exact chiral symmetry on the lattice for the first time. It was revealed that the sea quark gives only a small contribution to the nucleon sigma term.

In this study, we analyze the data of the nucleon mass obtained from a 2+1-flavor QCD simulation employing the overlap fermion [9]. We employ the chiral perturbation theory (ChPT) analysis for the nucleon mass data and calculate the sigma term from its up and down quark mass dependence. To calculate the strange quark mass dependence, we use the reweighting technique for the strange quark mass. Our study with exact chiral symmetry avoids the contamination due to a significant lattice artifact so that it provides a reliable calculation of the strange quark content.

\section{Lattice simulations}

We make an analysis of the nucleon mass obtained on 2+1-flavor QCD configurations generated with dynamical overlap fermions. Our simulations are performed on a $16^{3} \times 48$ lattice with a trivial topological sector $Q=0$. For the gluon part, the Iwasaki action is used at $\beta=2.30$ together with unphysical heavy Wilson fermions and associated twisted-mass ghosts [10] introduced to suppress unphysical near-zero modes of $H_{W}\left(-m_{0}\right)$. The lattice spacing $a=0.108 \mathrm{fm}\left(a^{-1}=1.83\right.$ $\mathrm{GeV}$ ) is determined through the Sommer scale $r_{0}=0.49 \mathrm{fm}$ of the static quark potential. We take two different strange sea quark masses $a m_{s}=0.080$ and 0.100 with five values of degenerate up and down quark masses (for $a m_{s}=0.080, a m_{\text {sea }}=0.015,0.025,0.035,0.050,0.080$ and for $\left.a m_{s}=0.100, a m_{\text {sea }}=0.015,0.025,0.035,0.050,0.100\right)$. We accumulate about 2,500 trajectories for each combination of sea quark masses; the calculation of the nucleon mass is carried out at every five trajectories, thus we have 500 samples for each sea quark masses. In order to improve the 

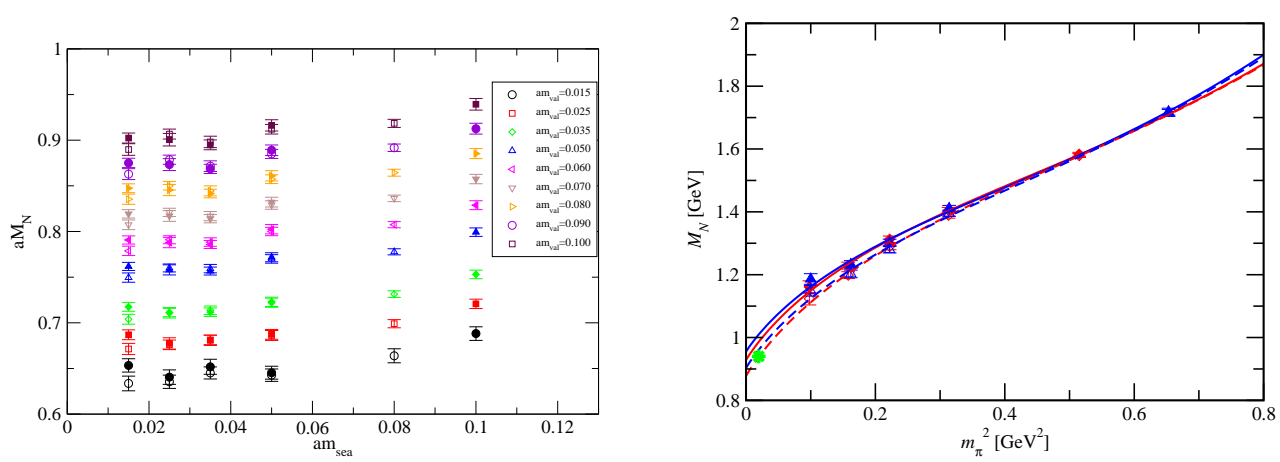

Figure 1: (Left) The nucleon mass data for the partially quenched data points. The empty and full symbols show the fit results for $a m_{s}=0.080$ and $a m_{s}=0.100$, respectively. (Right) Chiral fit of the lattice raw data(full symbols) and the finite size corrected data(empty symbols). The solid and dashed curve represent the fits of raw and corrected data. For a reference, we also show the experimental values of the nucleon mass (star).

statistical accuracy, we use the low-mode averaging technique. The two-point function made of the low-lying modes of the Dirac operator is averaged over different times slices with 80 chiral pairs of the low modes. For the quark propagator, we take a smeared-local two-point correlator and fit the data with a single exponential function after averaging over forward and backward propagating states in time. The statistical error is estimated using the starndard jackknife method with a bin size of 10 samples. We take the valence quark masses $a m_{v a l}=0.015,0.025,0.035,0.050,0.060$, $0.070,0.080,0.090$ and 0.100 . All the plots of the nucleon mass spectrums are shown in the left panel of Fig. 1 .

The matrix element defining the nucleon sigma term can be related to the quark mass dependence of the nucleon mass using the Feynman-Hellman theorem. For the strange sea quark, the matrix element is represented by the following relation as $\frac{\partial M_{N}}{\partial m_{\mathrm{s}}}=\langle N|\bar{s} s| N\rangle_{\text {disc. The subscript "disc" }}$ on the expectation value indicates that only the disconnected quark line contractions are evaluated. In the present study we exploit this indirect method to extract the matrix elements corresponding to the nucleon sigma term.

Another possible method is to directly calculate the matrix element from three-point functions with an insertion of the scalar density operator $(\bar{u} u+\bar{d} d)(x)$ or $\bar{s} s(x)$. We note that the indirect method gives mathematically equivalent results to those by the direct method including lattice artifacts. Numerical differences could arise only in the statistical error and the systematic uncertainties due to the fit ansatz.

\section{Analysis of the nucleon mass using chiral perturbation theory}

We carry out a fit with the SU(2) NLO ChPT for the baryon,

$$
M_{N}=M_{0}-4 c_{1} m_{\pi}^{2}-\frac{3 g_{A}^{2}}{32 \pi f_{\pi}^{2}} m_{\pi}^{3}+e_{1}^{r}(\mu) m_{\pi}^{4}
$$

where $M_{0}$ is the nucleon mass in the chiral limit and $f_{\pi}$ is the pion decay constant fixed at its physical value $92.4 \mathrm{MeV}$ and the constant $g_{A}$ describes the nucleon axial-vector coupling. We fix 


\begin{tabular}{cccc}
\hline & \multicolumn{2}{c}{ without FVCs } & with FVCs \\
& 4 points & 5 points & 5 points \\
\hline$a m_{s}=0.080$ & $50.0(4.5)$ & $48.2(3.0)$ & $51.1(3.0)$ \\
$a m_{s}=0.100$ & $51.3(4.1)$ & $45.5(3.0)$ & $48.2(3.0)$ \\
\hline
\end{tabular}

Table 1: Nucleon sigma term $\sigma_{\pi N}[\mathrm{MeV}]$ with and without the finite volume corrections (FVCs). The error is statistical only.

$g_{A}$ to its experimental value $g_{A}=1.267$. We use the pion mass obtained from the calculation of the meson spectra. Since our lattice volume is not large enough, the finite size effects must be taken into account especially in the data at lightest quark mass. To estimate possible systematic error, we attempt the fit with and without lightest sea quark mass (4 points and 5 points, respectively). We also correct the finite volume effect based on the NLO ChPT formula. The nucleon mass difference in the finite volume with box size $L$ and infinite volume $\delta M_{N}(L)$ is calculated within the ChPT. We use the asymptotic formula [11] to evaluate the magnitude of the finite volume effect as

$$
\delta M_{N}(L)=\left(\frac{9 g_{A}^{2} m_{\pi}^{2}}{16 \pi f_{\pi}^{2}}+\frac{4 g_{\Delta N}^{2} m_{\pi}^{\frac{5}{2}}}{(2 \pi)^{\frac{3}{2}} \Delta L^{\frac{1}{2}}}\right) \frac{1}{L} \exp \left(-m_{\pi} L\right),
$$

where $\Delta$ is the $\Delta$-nucleon mass splitting, and the coupling $g_{\Delta N}$ is decuplet-octet-axial coupling. We use the phenomenological estimates $g_{\Delta N}=1.5$ and $\Delta=300 \mathrm{MeV}$.

The chiral fit is made for the corrected five data points using the formula (3.1). The results are shown in the right panel of Fig. 1. After correcting the finite volume effect, there are $6 \%$ decrease in $M_{0}$ and 5\% increase in the magnitude of the slope $\left|c_{1}\right|$. Finally we obtain the nucleon sigma terms by estimating the pion mass dependence of the nucleon mass at the physical pion mass. The results are shown in Table 1. We observe that both the fit results at $a m_{s}=0.080$ and $a m_{s}=0.100$ without lightest points are consistent each other. These results are also consistent with our previous results for two-flavor simulations. We quote our best value of the sigma term as a fit result of 4 points fit $\left(a m_{s}=0.080\right.$, FVCs not included). We obtain $\sigma_{\pi N}=50.0(4.5)$. The finite volume correction of the sigma term amounts to about $6 \%$. We note that a sizable chiral extrapolation error should also be added after comparing NLO and NNLO in ChPT as found in our previous analysis with two-flavor QCD.

\section{Reweighting the strange quark mass}

In order to study the strange quark mass dependence of the nucleon, we utilize the reweighting technique [12, 13]. Using this method we can calculate any physical observables at a slightly different strange quark mass $\left(m_{s}^{\prime}\right)$ from the configurations $(U)$ generated at a certain value of strange quark mass $\left(m_{s}\right)$ as

$$
\langle\mathscr{O}\rangle_{m_{s}^{\prime}}=\frac{\left\langle\mathscr{O}\left(U, D\left(m_{s}^{\prime}\right)\right) w\left(m_{s}^{\prime}, m_{s}\right)\right\rangle}{\left\langle w\left(m_{s}^{\prime}, m_{s}\right)\right\rangle}
$$

where $D$ is overlap Dirac operator and we introduce the reweighting factor $w\left(m_{s}^{\prime}, m_{s}\right)$ defined as $w\left(m_{s}^{\prime}, m_{s}\right)=\operatorname{det}\left[\frac{D\left(m_{s}^{\prime}\right)}{D\left(m_{s}\right)}\right]$. We calculate the reweighting factor by decomposing it into the low and 


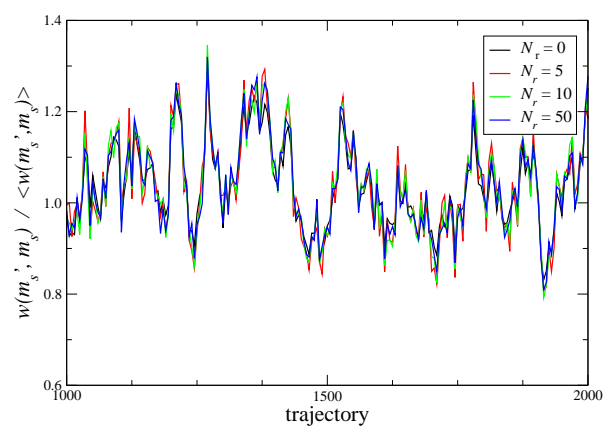

Figure 2: The fluctuations of the reweighting factor. The plot shows the noise number dependence of the fluctuations at $a m_{v a l}=a m_{\text {sea }}=0.050$ with strange quark masses $a m_{s}^{\prime}=0.075, a m_{s}=0.080$.

high-mode contributions. A former contribution is obtained exactly by the 80 pairs of low-lying eigenvalues of the Dirac operator. For high-mode, we use the improved stochastic estimator

$$
\begin{gathered}
w_{\text {high }}\left(m_{s}^{\prime}, m_{s}\right)=\sqrt{\frac{1}{N_{r}} \sum_{r=1}^{N_{r}} e^{-\frac{1}{2}\left(\bar{P} \xi_{r}\right)^{\dagger}(\Omega-1) \bar{P} \xi_{r}},} \\
\Omega \equiv D\left(m_{s}\right)^{\dagger}\left\{D\left(m_{s}^{\prime}\right)^{-1}\right\}^{\dagger} D\left(m_{s}^{\prime}\right)^{-1} D\left(m_{s}\right),
\end{gathered}
$$

where $\xi_{r}$ are the ensembles of pseudo-fermion fields ( $N_{r}$ : number of noise vector), and each are generated with Gaussian probability. Here we also introduce the projection operators $P$ and $\bar{P}$ to project the eigenspaces of $\xi$ into low and high-modes, respectively.

Fig. 2 shows the reweighting factor with $a m_{s}^{\prime}=0.075$ and $a m_{s}=0.080$ for each gauge configuration. It is evaluated with different number of noise vector $N_{r}=0,5,10$ and 50. We find that the statistical error of the reweighting factor is very small compared to that from the fluctuation of the gauge configurations. We also find that the contribution from high-mode is almost independent of the gauge configuration and the configuration dependence is almost completely dictated by the low-mode contribution. These properties are universal for entire region of up and down quark masses.

In order to estimate the strange quark mass dependence of the nucleon mass, we evaluate the nucleon masses at 10 different strange quark mass for each configurations. That covers the strange quark mass range $a m_{s}^{\prime}=0.0775-0.0825$ for $a m_{s}=0.080$ and $a m_{s}^{\prime}=0.0975-0.1025$ for $a m_{s}=0.100$. To compute the high-mode contributions for several $m_{s}^{\prime}$ at once, we use the multishift solver for the Dirac operator. We take five random noises for the noisy estimator. Thus the nucleon mass for each different strange quark masses is calculated. We find small strange quark mass dependence of the nucleon mass and its statistical error does not grow for entire region of the strange quark mass we take.

\section{Extraction of the strange quark content}

To extract the strange quark content $\langle N|\bar{s} s| N\rangle$, we fit the nucleon mass by a linear function of strange quark mass. Then the coefficient of the linear term corresponds to the strange quark content of the nucleon. We use 11 data points for the fit including the unreweighted point at each sea and 


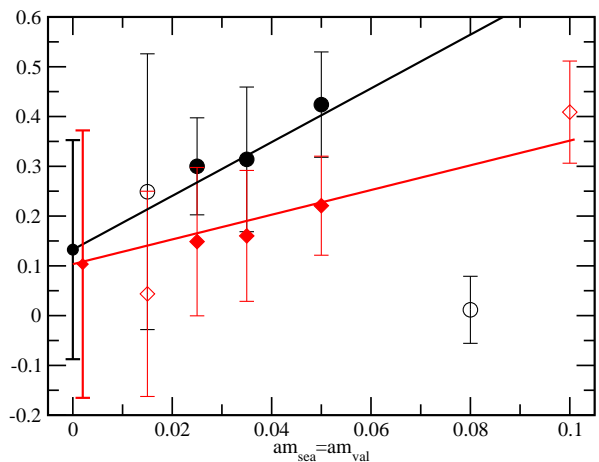

Figure 3: The chiral extrapolation fit for the strange quark content for $m_{s}=0.080$ (black) and $m_{s}=$ 0.100 (red). The plot shows only the data at unitary point. The linear line shows the fit functions for unitary point. For a reference, the empty symbols show the data not used in the fit.

\begin{tabular}{ccccccc}
\hline$a m_{\text {sea }}=a m_{\text {val }}$ & 0.015 & 0.025 & 0.035 & 0.050 & 0.080 & 0.100 \\
\hline$a m_{s}=0.080$ & $0.25(28)$ & $0.30(10)$ & $0.31(15)$ & $0.42(11)$ & $0.01(7)$ & - \\
$a m_{s}=0.100$ & $0.04(20)$ & $0.15(15)$ & $0.16(13)$ & $0.22(10)$ & - & $0.41(10)$ \\
\hline
\end{tabular}

Table 2: The strange content of the nucleon at the unitary points.

valence quark masses. We obtain the non-zero signals of the strange content at each data point with $50-100 \%$ statistical error. The results for the unitary points are shown in Table 2 . To estimate the physical values of the strange content, we need to carry out a chiral extrapolation. In order to improve the accuracy, we use partially quenched data point. We take the fit ranges as $a m_{\text {sea }}=0.025$ 0.050 and $a m_{v a l}=0.025-0.050$ to avoid large finite volume corrections and possible higher order corrections. Its chiral behavior is described by a derivative of the nucleon mass formula in SU(3) ChPT in terms of the strange quark mass. We note that the non-analytic behavior of the light up and down quark masses does not appear up to NNLO corrections. Therefore we safely use the polynomial function of the up and down quark masses. We use a simple fit function

$$
\frac{d M_{N}}{d m_{s}}=a_{1}+a_{2} m_{\text {sea }}+a_{3} m_{v a l} .
$$

The fit results are shown in Fig. 3. The data are reasonably fitted by the formula (5.1). Although our results at chiral limit have about $100 \%$ errors, it shows that the physical result of the strange quark content is very tiny. We estimate the renormalization group invariant quantity $f_{T_{s}}$ as

$$
f_{T_{s}}=\left\{\begin{array}{ll}
0.020(34) & \left(a m_{s}=0.080\right) \\
0.019(53) & \left(a m_{s}=0.100\right)
\end{array},\right.
$$

where the error is statistical. There may be some systematic uncertainties coming from the finite volume corrections and chiral extrapolations. Both results are consistent each other, we find the strange quark mass dependence of this quantity is very small. Using two results of the sigma term obtained in Sec. 3 and $f_{T_{s}}(=0.020)$, we obtain $y \sim 0.03$. These results are comparable to our previous two-flavor analysis [6]. We also conclude that the upper bound on $f_{T_{s}}$ is given by $\left|f_{T_{s}}\right| \leq 0.08$ at $1 \sigma$ level. 


\section{Summary}

We calculated the nucleon mass and sigma term in 2+1-flavor QCD simulation on the lattice with exact chiral symmetry. To evaluate the strange quark content of the nucleon, we used the reweighting technique for the strange quark mass, worked very well for studying the strange quark mass dependence. Although our results have large statistical uncertainty, we conclude that the strange quark content of nucleon is tiny compared to its up and down quark contributions.

The main numerical calculations were performed on IBM System Blue Bene Solution at High Energy Accelator Organization (KEK) under support of its Large Scale Simulation Program (No.09-05). We also used NEC SX-8 at Yukawa Institute for Theoretical Physics (YITP), Kyoto University and at Research Center for Nuclear Physics (RCNP), Osaka University. This work is supported in part by the Grant-in-Aid of the Ministry of Education (Nos. 21.897, 19540286, 19740160, 20105001, 20105002, 20105003, 20105005, 20340047, 21674002, 21684013). The work of HF was supported by the Global COE program of Nagoya University "QFPU” from JSPS and MEXT of Japan.

\section{References}

[1] K. Griest, Phys. Rev. Lett. 61, 666 (1988). K. Griest, Phys. Rev. D 38, 2357 (1988) [Erratum-ibid. D 39, 3802 (1989)].

[2] J. Ellis, K. A. Olive and C. Savage, Phys. Rev. D 77, 065026 (2008) [arXiv:0801.3656 [hep-ph]].

[3] M. Fukugita et al., Phys. Rev. D51 (1995) 5319, hep-lat/9408002,

[4] S.J. Dong, J.F. Lagae and K.F. Liu, Phys. Rev. D54 (1996) 5496, hep-ph/9602259,

[5] SESAM, S. Gusken et al., Phys. Rev. D59 (1999) 054504, hep-lat/9809066,

[6] H. Ohki et al. [JLQCD Collaboration], Phys. Rev. D 78, 054502 (2008) [arXiv:0806.4744 [hep-lat]].

[7] R. D. Young and A. W. Thomas, arXiv:0901.3310 [hep-lat].

[8] C. Michael, C. McNeile and D. Hepburn [UKQCD Collaboration], Nucl. Phys. Proc. Suppl. 106, 293 (2002) [arXiv:hep-lat/0109028].

[9] H. Matsufuru et al. (JLQCD and TWQCD collaborations), PoS LAT2008, 077 (2008)

[10] H. Fukaya, S. Hashimoto, K. I. Ishikawa, T. Kaneko, H. Matsufuru, T. Onogi and N. Yamada [JLQCD Collaboration], [arXiv:hep-lat/0607020].

[11] S. R. Beane, Phys. Rev. D 70, 034507 (2004) [arXiv:hep-lat/0403015].

[12] A. Hasenfratz, R. Hoffmann and S. Schaefer, Phys. Rev. D 78, 014515 (2008) [arXiv:0805.2369 [hep-lat]].

[13] T. DeGrand, Phys. Rev. D 78, 117504 (2008) [arXiv:0810.0676 [hep-lat]]. 